\title{
Obesity and sleep apnea
}

\section{Opinion}

Obesity can be defined as a state in which there is excess amount of fat distributed throughout the body. It is basically a chronic condition where important factors play a major role like environment, eating behaviour, physical inactivity, genetics/hereditary and psychosocial factors. Although it is a well-known fact that certain amount of fat is required for heat insulation, storing energy, shock absorption, etc. but beyond a certain level, it leads to obesity. Often, obesity is defined in terms of BMI (Body Mass Index), a person having BMI $\geq 30 \mathrm{~kg} / \mathrm{m}^{2}$ falls in the category of obesity where BMI $\geq 25 \mathrm{~kg} / \mathrm{m}^{2}$ indicates that the person is overweight.

People who are obese also suffer in their sleep, usually they have shorter sleep duration as compared to people of non-obese category. The quality as well as quantity both are affected. Obesity becomes a major risk factor for development of a type of sleep disorder called Obstructive Sleep Apnea (OSA) and progression. OSA is defined as intermittent or complete upper airway collapse while a person is in sleep, which leads to fragmentation in sleep, decreased levels of bloody oxygen (also known as hypoxemia), increased levels of carbon dioxide $\left(\mathrm{CO}_{2}\right.$ - hypercapnia), intrathoracic pressure oscillations and increased sympathetic activity. The diagnosis and severity of this disease is based on Apnea Hypoapnea Index (AHI). AHI between 5 and 15 is termed as mild OSA, between 15 and 30 is called as moderate OSA and $>30$ is known as severe OSA. Since the awareness of this sleep disorder is less amongst the population, $80 \%$ of the OSA cases go undiagnosed. ${ }^{1-4}$

The prevalence of OSA in obese person is almost twice that of normal weight person. An adult having mild OSA who gains even $10 \%$ of their baseline weight are at six-fold increased risk of developing or progressing OSA. Since in OSA there are chances that obesity worsens the condition due to fat deposition at specific sites. A linear correlation has been observed between the two. Due to fat deposition in the tissues of upper airway, it leads to smaller lumen and increased chances of collapsibility of the airway, further developing apnea. Also, the fat which deposits around abdomen reduces chest compliance and functional residual capacity in lungs leading to slight increase in the oxygen demand. Visceral obesity is the most common found in people suffering from OSA, the chances of OSA is seen higher in men as compared to women $(2 \mathrm{x})$.

Patients with OSA are at an increased risk of death, hence, a thorough body assessment to monitor the impact of weight on upper airway which also includes the metabolic and anthropometry check becomes extremely important.

Weight gain doesn't occur in a day or two, it takes time for a person to gain weight and is influenced by lifestyle factors such as lack of sleep, excessive calorie intake, hereditary and sedentary lifestyle. Lack of sleep and obesity both can contribute to depression. Also, short sleep duration and inadequate dietary intake can cause hormonal imbalance. One of the great examples, is decrease in melatonin hormone, which leads to changes in circadian rhythm contributing
Volume 9 Issue 3 - 2019

\author{
Saman Zaman \\ Nutritionist, India \\ Correspondence: Saman Zaman, Nutritionist, Tel \\ 919953250295, India, Email samanzaman03@gmail.com \\ Received: May 15, 2 019| Published: May 27, 2019
}

to weight gain and metabolic syndrome. Changes are also seen in the levels of hormone insulin and leptin. Usually an obese person develops resistance to both the above hormones which decreases the food need and increases energy metabolism. Another hormone called Ghrelin released by stomach stimulates the appetite and gets affected by disturbances in sleep. These modified levels seen in chronic short sleepers predispose obesity i.e. increase in the level of ghrelin and decrease in the level of leptin. Factors leading to obesity such a lack of sleep and excessive calorie intake becomes primary reason for developing diabetes and heart diseases. Due to low oxygen levels in the blood, the oxidative stress leads to excess generation of reactive oxygen species which can cause endothelial dysfunction and result in atherosclerosis.

Weight loss appears to be an effective therapy, especially in case of obesity where $>70 \%$ of them are suffering with OSA. Weight has shown positive results in reducing the severity and symptoms of OSA. Apart from this, weight loss also helps in reducing the levels of cholesterol, insulin resistance, leptin, inflammatory markers and endothelial functions or any other abnormalities related to OSA.

\section{Acknowledgments}

None.

\section{Conflicts of interest}

The authors declared there is no conflict of interest.

\section{References}

1. Romero-Corral A, Caples SM, Lopez-Jimenez F, et al. Interactions between Obesity and Obstructive Sleep apnea. Chest. 2010;137(3):711719.

2. Magdalena Dobrowolska, Izabella Dunin-Wilczynska, Iwona Mitura, et al. BMI in patients with obstructive sleep apnea. Currents issues in Pharmacy and Medical Sciences. 2015.

3. Joese Mauricio, Leandro Ortega, Alessandra Loli, et al. Evaluation of obstructive sleep apnea in obese patients scheduled for bariatric surgery. Acta Cirurgica Brasileira. 2013;28(4):317.

4. Jehan S, Zizi F, Pandi-Perumal SR, et al. Obstructive Sleep Apnea and Obesity: Implications for Public Health. Sleep Med Disord. 2017;1(4). 